\title{
Téoros
}

Revue de recherche en tourisme

\section{La vallée du Zat}

Un pays d'accueil touristique émergeant dans l'arrière-pays montagneux de Marrakech

\section{Ahmed Bellaoui}

Volume 24, numéro 1, printemps 2005

Maroc, réalités et défis

URI : https://id.erudit.org/iderudit/1071109ar

DOI : https://doi.org/10.7202/1071109ar

Aller au sommaire du numéro

Éditeur(s)

Université du Québec à Montréal

ISSN

0712-8657 (imprimé)

1923-2705 (numérique)

Découvrir la revue

Citer cet article

Bellaoui, A. (2005). La vallée du Zat : un pays d'accueil touristique émergeant dans l'arrière-pays montagneux de Marrakech. Téoros, 24(1), 42-47.

https://doi.org/10.7202/1071109ar d'utilisation que vous pouvez consulter en ligne.

https://apropos.erudit.org/fr/usagers/politique-dutilisation/ 


\section{La vallée du Zat}

\section{Un pays d'accueil touristique émergeant dans l'arrière-pays montagneux de Marrakech}

\begin{abstract}
Ahmed Bellaoui
Située à moins d'une heure de la ville rose de Marrakech, sur la route de Ouarzazate, la vallée du Zat, comparée à celles de l'Ourika, du Rherhaîa et du Nfis, commence à peine à se faire connaitre des touristes aussi bien marocains qu'étrangers. Découvertes et mises en vedette puis commercialisées et fortement médiatisées depuis les premières années du protectorat, ces dernières ne diffèrent pourtant en rien de la première, sinon par leur proximité du massif du Toubkal.
\end{abstract}

En effet, celui-ci, exploré dès le début des années 1920, n'a cessé d'attirer les amoureux, de plus en plus nombreux, de l'escalade et du ski d'abord, de la randonnée, de la nature et de l'écotourisme ensuite (Bellaoui, 2004), grâce beaucoup à la proximité de Marrakech, en tant que pôle touristique de renommée internationale; les professionnels du tourisme national et étranger ont fait le reste. Toutes les autres vallées du versant nord du Haut Atlas de Marrakech, pourtant tout aussi attrayantes que celles des abords immédiats du massif du Toubkal, ont vécu quasiment inconnues, à l'ombre de ces dernières.

Se situant dans le prolongement du Guide Alpin de la Montagne Marocaine (Dresch et De Lepiney, 1938), les guides consacrés à Marrakech et sa région se contentent par ailleurs de ressasser les « pistes » tracées par ce dernier. Mieux encore, même le Guide de renseignements pratiques, édité par l'Office national marocain du Tourisme (1995) à l'occasion de la Grande Traversée des Atlas Marocains (GTAM), n'en dit mot. Autant dire que la vallée du Zat ne semble pas avoir intéressé les éditeurs des guides touristiques et, à travers eux, les professionnels du tourisme.
Pourtant, à l'instar des autres vallées, la vallée du Zat possède un nombre important d'atouts susceptibles d'en faire une destination touristique tout aussi attractive que l'Ourika ou le Rherhaîa, par exemple. Quels sont ces atouts ? Quel sont les impacts à la fois sur les pratiques, les flux et les infrastructures touristiques en place? Et comment la vallée du Zat réagit-elle à sa récente mise en tourisme? Des questions auxquelles cet article tentera d'apporter quelques éléments de réponse.

\section{Des paysages naturels et humains authentiques et encore fortement bien conservés et dont l'appréciation interpelle tous les sens}

La vallée du Zat offre au moins trois catégories de paysages:

- les paysages des cuvettes et des bas plateaux de la zone des avant-monts,

- les paysages de la moyenne montagne,

- les paysages de la haute montagne ou du Haut Zat.

\section{Les paysages des cuvettes et des bas plateaux de la zone des avant-monts}

Après avoir roulé prés d'une demi-heure sur la plaine du Haouz en direction de Ouarzazate, le voyageur, en provenance de Marrakech, aborde la vallée du Zat à hauteur de la petite ville d'Ait Ourir et de son souk hebdomadaire dit Tlat n'Imi $n$ Zat. L'arrivée sur le versant nord du Haut Atlas de Marrakech se fait par une zone de contact, appelée Dir (Bellaoui, 1994), où dominent des reliefs de faible altitude (de 1000 à 1500 mètres) composés essentiellement de crêts (Jbel Adendim), de bas plateaux (Tasghimout) et de cuvettes (Ait
Ziyad). Encore largement sous l'influence desséchante de la plaine du Haouz et parce que très fortement affectée par l'érosion anthropique, cette zone de contact, large de près de 15 à 20 kilomètres, offre des paysages assez contrastés, parmi lesquels :

\footnotetext{
- les paysages verdoyants des oliveraies de l'Imi n Zat (Ait Ourir, Agadir n Ait Lahcen, Taferiat, Imi n Reg) et de la cuvette des Ait Ziyad d'un côté, les pineraies du Jbel Adendim et le plateau de Tasghimout de l'autre ;

- les paysages jaune paille des crêts et des plateaux totalement déboisés des Ait Ziyad, essentiellement voués à la céréaliculture et à l'élevage extensif.
}

Les douars, dont l'implantation est fortement liée à la présence de l'eau, soit s'agglomèrent au pied des crêtes et des plateaux, soit s'étirent le long des berges de l'oued. Groupés pour la plupart et relativement proches les uns des autres, ces villages rappellent ceux de la plaine aussi bien par l'emploi du pisé et l'existence de grandes ouvertures que par la prédominance de grandes demeures à un seul niveau.

\section{Les paysages de la moyenne montagne}

La montagne proprement dite commence avec l'arrivée au pied des flancs nord et escarpés des hauts plateaux permo-triasiques qui forment, de part et d'autre de la vallée du Zat, la moyenne montagne dont l'altitude varie de 1500 à 2500 mètres. Là, la vallée commence à se resserrer pour donner lieu à un encaissement qui devient de plus en plus important en direction de l'amont, vers le sud. Par endroit, celleci s'élargit, cependant en de petits bassins intra-montagneux aux sols relativement ri- 
ches qui accrochent à la fois par l'intensité des cultures, la densité de l'habitat et la luxuriance de la végétation. Celui de Larbaa Tighdouine, situé à près de 10 kilomètres à l'intérieur de la moyenne montagne sur la RP $31 d$, offre une multitude de coloris.

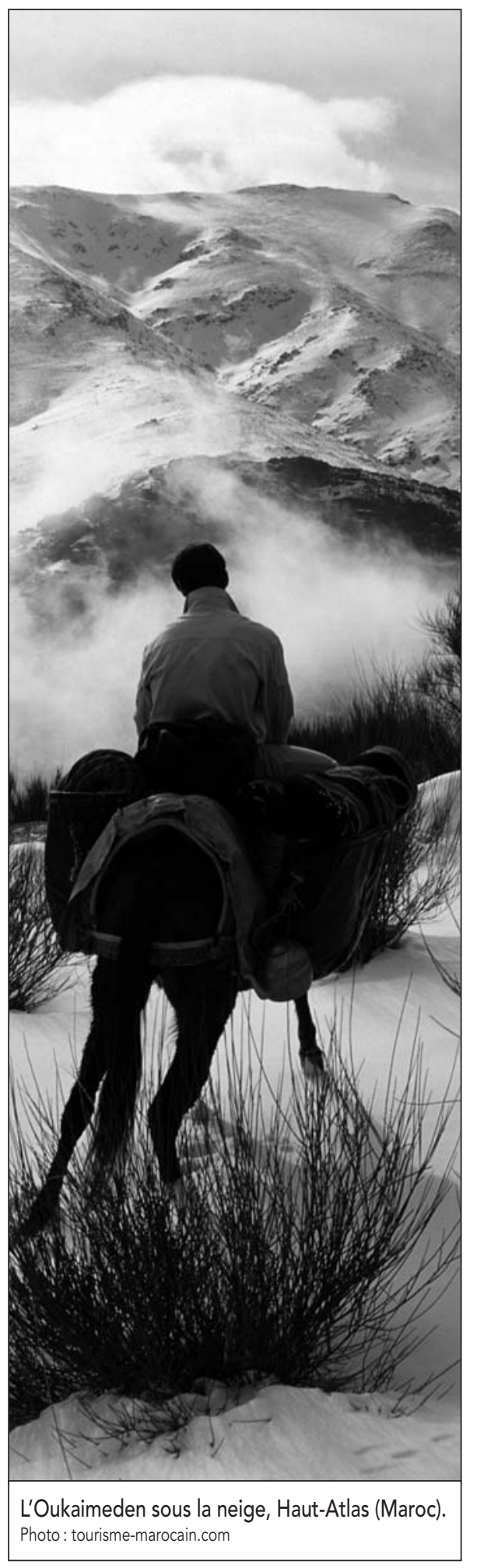

D'un vert franc et vif au fond de la vallée en rapport avec les cultures (verveine, tomates, maïs, blé, pomme de terre), la végétation se ternit en altitude et s'uniformise en de nombreux buissons sombres faits de cistes, de lentisques, d'oléastres et de caroubiers. Des taches roses de lauriers auxquelles se mêlent, par endroit, les massifs verdâtres de peupliers et d'oliviers, ornent le fond de la vallée. Sur les versants, des deux côtés de la rivière, la dégradation de la végétation laisse apparaître les formations argilo-gréseuses, le long des ruisseaux et des ravins. Le sens de la vue se trouve ainsi fortement stimulé, surtout par temps clair, par cette abondance de couleurs dès l'entrée en moyenne montagne.

Fortement influencée par l'altitude, la zone peut recevoir d'importantes quantités de pluie (près de 500 millimètres en moyenne par an), voire de neige au-delà de 1800 à 2000 mètres, donnant ainsi naissance, en particulier sur les versants les mieux exposés aux influences océaniques, soit à de magnifiques massifs forestiers où se mêlent thuya, chêne et genévrier oxycèdre, soit à d'importants terrains de parcours sur les hauts plateaux permo-triasiques du Tissiyi, de l'Iferwane et du Yagour. Autant d'atouts auxquels la société chleuh haut atlasique ne pouvait rester indifférente, la moyenne montagne se trouvant être la partie la plus fortement humanisée et la mieux aménagée du versant nord du Haut Atlas de Marrakech (Bellaoui, 1992).

En effet, les douars, ici très nombreux, sont établis soit à mi-pente sur les replats qui rompent la raideur des flancs des hauts plateaux, soit le long de l'oued sur les hautes terrasses fluviales, bien à l'abri des crues, très dévastatrices par ailleurs. Les habitations, quant à elles, sont construites, pour la plupart, en grès rose dont la pierre est taillée avec art par des maîtres-maçons locaux dont le savoir-faire mérite d'être préservé. Partout les maisons sont formées d'un rez-de-chaussée (qui sert d'étable) et d'un étage dont le toit sert à faire sécher les épis de maïs pendant les journées d'automne et à se rafraîchir pendant les chaudes nuits d'été.

Nul n'est besoin de dire qu'à cet important enracinement par l'habitat répond une appropriation tout aussi poussée de l'espace agricole par le biais d'un système de culture autant ingénieux qu'efficace. Construits au prix d'un effort quasiment titanesque, les champs, le plus souvent en terrasses savamment alignées les unes au-dessus des autres selon les courbes de niveau, portent des cultures qui font place, de plus en plus, à l'arboriculture (oliviers surtout) et au maraîchage (pommes de terre, navets, tomates). Partout, les céréales reculent (excepté le maïs et le millet), sauf sur les versants les mieux arrosés où elles constituent, encore, l'essentiel des cultures dites «bour » ou non irriguées (Bellaoui, 1990).

L'importance de l'irrigué, surtout le long de l'oued Zat et sur les replats de mi-versant, se traduit par l'existence d'une série d'aménagements hydrauliques inséparables des paysages haut atlasiques : les targa (canaux d'irrigation), les tifarwin (bassins de retenue), et les ouggoug (petits barrages de dérivation). Reliés soit aux ouggoug construits au fil de l'eau, soit aux tifarwin aménagés au-dessus des villages, les targa, soulignés généralement par une double haie d'arbres ou d'arbustes, vivifient les minuscules parcelles qui forment, par endroit, de remarquables « petits périmètres irrigués ", autre spécificité de la moyenne montagne.

Au-delà de Larbaa, la vallée se resserre de façon impressionnante. Large d'à peine quelques dizaines de mètres, celle-ci est fortement dominée par le haut plateau permotriasique du Yagour qui forme, par endroit, de gigantesques falaises de couleur rouge, comme chez les Ait Ouagstit, par exemple. La présence de l'homme est réduite ici à sa moindre expression: à peine l'aménagement de quelques prés le long de l'oued!!! Toute son action se trouve portée en altitude, le plus souvent, le long des vallées affluentes du Zat qui donnent naissance à de petits bassins de vie relativement importants tels ceux des fractions des Ait Tighdouine et des Ait Ouagstit, sur la rive droite de l'oued, et des Ait Inzal de la montagne, sur la rive gauche.

\section{Les paysages de la haute montagne ou du Haut Zat}

La vallée, dont les eaux limpides regorgent de truites, se transforme surtout à partir du lieudit Imirguen, en un étroit couloir dont le resserrement croît en direction du Haut Zat, donnant lieu à de véritables gorges dont les parois abritent quelques singes migrateurs, comme à hauteur des douars Taliwin et Tiniyiguen, accrochés au Jbel Meltsen, côté sud. 
Le paysage est, ici, beaucoup plus austère que dans la moyenne montagne et les teintes sont beaucoup plus atténuées. Le rouge-ocre des formations argilo-gréseuses est remplacé par les teintes plus sombres des formations granitiques et rhyolitiques. Cette austérité, que ne rompt que la blancheur immaculée de la neige hivernale, est accentuée par la raideur des versants et par l'encaissement de la vallée. Les reliefs, beaucoup plus aigus et plus élevés, donnent à cette zone un côté plus agressif et moins accueillant, mais fortement prisé par le bouquetin de l'Atlas et le mouflon. Cette impression est d'autant plus forte que le granite et les rhyolites résistent mieux à l'érosion et que les sommets, en s'imbriquant les uns dans les autres, obligent à rester dans le fond de la vallée au milieu des saules pourpres, des joncs et des noyers.

Sur les deux rives de l'oued, la végétation est à la fois peu diversifiée, peu abondante et largement dominée par le chêne vert et le genévrier thurifère avant de céder la place aux plantes à coussinet sur les hauts sommets. Fortement affectée par la sécheresse des dernières années, par la dent de la chèvre, mais aussi et surtout par la hache du berger et de la ménagère, la végétation souffre scandaleusement de désertification. L'habitat, dans cette section de l'oued Zat, se résout à éviter entièrement le fond de la vallée et à s'organiser à mi-versant à l'occasion de la moindre rupture de pente. Très groupées, les habitations, disposées en escalier, s'ouvrent toutes, telles de gigantesques nids d'abeilles, sur la rivière et possèdent à l'étage une tasdiht (véranda) utilisée pour le séchage des cultures et pour se réchauffer au soleil pendant les froides journées d'hiver.

Les matériaux utilisés ici sont la pierre et, secondairement, le pisé. À base essentiellement de granite et de rhyolite particulièrement résistants, la pierre se prête moins bien à la taille que le grès rose; d'où l'austérité des constructions dont l'architecture est ici plus modeste et moins bien soignée qu'en moyenne montagne.

Tout aussi différente, I'utilisation du sol, très rare d'ailleurs, est ici largement dominée par des cultures et des arbres fruitiers : noyer, figuier, pêcher, iris, pommes de terre, maïs, orge et, récemment, pommier. Façonnés quasiment à même la roche, les champs, sous forme de minuscules terrasses d'un vert éclatant, s'organisent en escaliers en

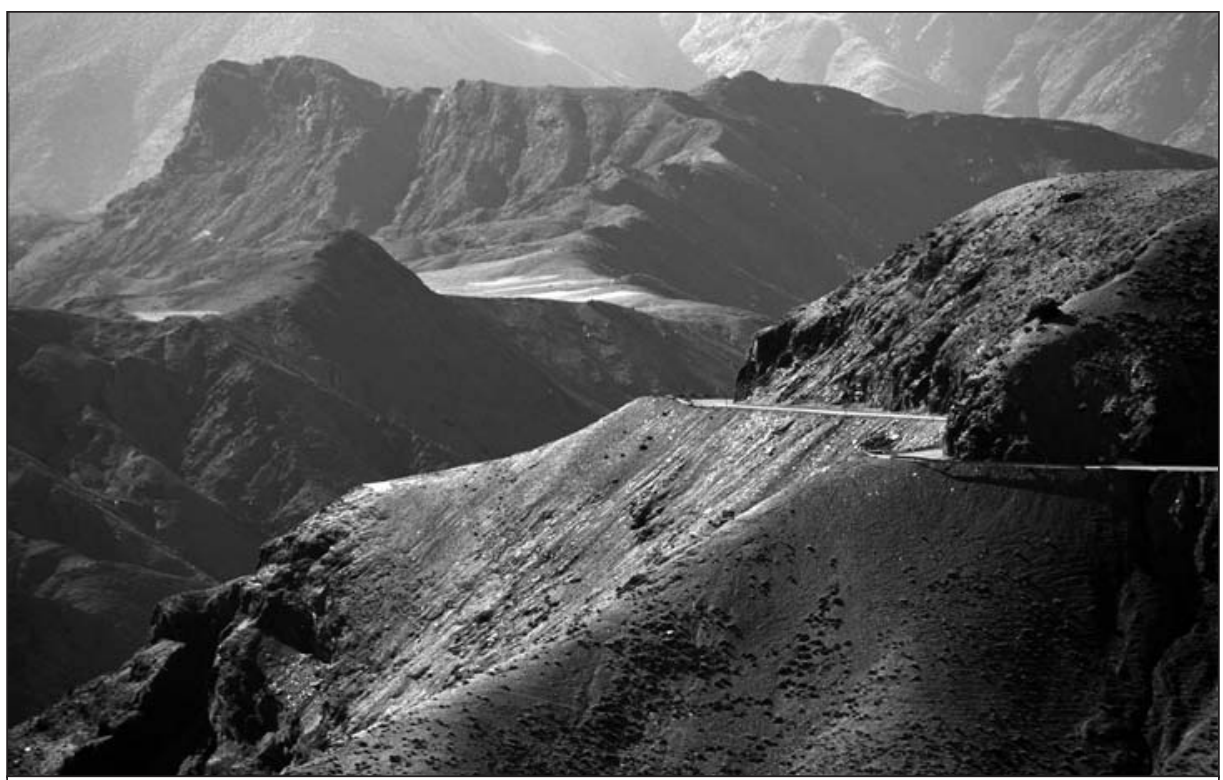

Route du col du Tichka, Haut-Atlas (Maroc).

photo : tourisme-marocain.com

contrebas des douars, accrochés au flanc sud des montagnes de l'lkis et du Meltsen.

La modicité des cultures est, ici, compensée par la place réservée à l'élevage, surtout caprin, qui bénéficie de l'existence de grands espaces collectifs de pâturage qui s'étendent jusqu'au sommet des crêtes de la haute montagne et, au-delà, jusqu'au Yagour. L'importance de ces parcours collectifs se justifie par l'existence, à cette altitude, d'une strate herbacée aux couleurs et aux odeurs infinies, donnant lieu à des pâturages (Igdlane, sing. Agdal) qui font l'originalité de ces étendues qui forment, en contrebas du majestueux Meltsen (3595 mètres), les plateaux permo-triasiques de la moyenne montagne.

Ces plateaux - le Yagour, et secondairement, le Tissiyi et l'Iferwane - forment de hauts lieux de pastoralisme, collectivement appropriés et exploités par la quasi-totalité des douars de la vallée, dans le cadre d'une "petite transhumance » née des rapports «vallée - hauts plateaux " que nous avons qualifiée, ailleurs, de " complémentarité horizontale " (Bellaoui, 1989). Ces derniers, riches en herbes de toutes sortes et interdits au pâturage, généralement, d'avril à juin, sont parsemés d'azibs (bergeries) qui reçoivent, dès le mois de juillet, un nombre impressionnant de chèvres et de moutons. Par endroit, ces azibs tendent, de nos jours, à se transformer en habitations en dur où logent les bergers et leur famille, pendant les mois d'été.
Ainsi donc, d'un bout l'autre de la vallée du Zat, le promeneur ne cessera de découvrir et d'apprécier la diversité et la variété des paysages naturels et humains, des couleurs, des lumières, des odeurs et des sons. C'est cette diversité au sein d'un espace relativement réduit (moins de 500 kilomètres carrés de superficie), en associant les plaisirs des yeux, du nez et de l'oreille, qui fait l'originalité de la vallée du Zat. Une originalité qui se trouve, par ailleurs, renforcée par l'existence, toujours en matière de tourisme, d'autres atouts tout aussi importants que les paysages.

\section{Les autres atouts touristiques de la vallée du Zat}

\section{La source de Sidi El Wafi : une eau minérale dont les effets thérapeutiques ont largement contribué à la mise en tourisme de la vallée du Zat}

Parmi les motivations qui ont contribué à la naissance du tourisme, la recherche du bien-être et, donc, de la santé du corps et de l'esprit, l'homme ayant, de tout temps, nourri une passion toute particulière pour l'art des soins corporels. La quête de la remise en forme par l'homme d'aujourd'hui fortement éprouvé par la vie combien stressante des agglomérations urbaines, en particulier, a largement contribué au développement du tourisme de santé. La découverte et l'exploitation de la source de Sidi El Wafi, en 
juillet 1990 à près d'une demi-heure de marche du chef-lieu de la commune de Tighdouine, ont largement contribué à la mise en tourisme de la vallée. Quasiment inconnue jusqu'alors, même des marrakchis pourtant très proches, la vallée du Zat, dont le nom est associé à celui de Laouina (source), est prise d'assaut par les touristes dont le nombre moyen a été estimé à près de 3500 par jour en 1990 (Boujrouf, 1994)!

Largement dominés par les curistes, les touristes, surtout nationaux, recherchent dans la vallée repos et fraîcheur. En effet, le site de la source de Sidi El Wafi est beau, pour ne pas dire enchanteur. Totalement camouflé sous les bois, le site est à la fois frais et ombragé : un site idéal pour les curistes, mais aussi pour les gens de la plaine du Haouz et de la ville de Marrakech, particulièrement sèches en été. La source de Sidi El Wafi offre par ailleurs aux visiteurs une gamme assez variée de produits : eau minérale, boues curatives et douches chaudes.

\section{Un patrimoine rupestre de haute valeur archéologique et culturelle au pied du Meltsen sur le plateau du Yagour}

À la différence des autres vallées du Haut Atlas de Marrakech, dont la participation parfois forte à l'histoire du pays a donné lieu à l'édification d'un nombre relativement important de monuments d'une grande valeur historique, la vallée du Zat ne possède aucun vestige de l'importance de la mosquée de Tinmel ou des kasbah de Tagoundaft et de Tazzert, par exemple. Sans doute parce que celle-ci, appartenant au territoire tribal des Mesfioua rebelles au Makhzen, n'a jamais connu l'avènement de personnalités politiques de grande renommée. Les chioukh's et les zaouias ne semblent jamais avoir étendu leur pouvoir au-delà des limites de la vallée; pour beaucoup d'entre eux, le commandement ne dépassait guère quelques douars ou, pour les plus puissants, une à deux fractions.

Mais la vallée du Zat possède, sur le plateau du Yagour, l'un des nombreux sites de gravures rupestres du Haut Atlas de Marrakech. Perché à près de 2700 mètres au-dessus des vallées de l'Ourika à l'ouest et du Zat à l'est, le plateau du Yagour semble avoir fixé, il y a près de 4000 ans, des pasteurs d'origine subsaharienne en quête de pâturages, chassés par la progression du désert.
En guise de témoignages, ces pasteurs ont gravé sur les dalles gréseuses du plateau des scènes entières de leur vie quotidienne, l'art rupestre ayant été le seul moyen d'expression à une époque où l'écriture n'était pas encore inventée. D'où l'importance de ces images gravées sur les grès dont la lecture et l'interprétation sont susceptibles de donner une meilleure connaissance du passé lointain de la vallée du Zat, en particulier, et du Maroc, en général.

Associant animaux sauvages, bœufs domestiqués, poignards, chars, hallebardes et hommes de grande taille, ces gravures se répartissent en deux sites distincts : le site de Warzazat au pied de l'Adrar n Meltsen et le site du plateau du Yagour proprement dit (Malhomme, 1959). Le site de Warzazat, auquel on accède par la piste dite du Yagour, est, sans aucun doute, le plus important. Plusieurs raisons à cela: d'abord, le nombre d'images qu'il renferme (de 1700 à 2000) ; leur concentration en un site facile à délimiter ; leur situation au pied du plus haut sommet de la vallée (le Meltsen) et à proximité du col de Tizi N'Rhellis qui permet d'accéder à la vallée voisine de l'Ourika. Le site du plateau du Yagour proprement dit, situé au-dessus et à droite du Tizi N'Rhellis qui le sépare du Meltsen, est beaucoup plus difficile à identifier. II est, en effet, beaucoup plus dispersé que celui de Warzazat, probablement en raison de l'étendue du plateau lui-même.

Mais aujourd'hui, ces gravures, dont la richesse patrimoniale est incontestable, sont gravement menacées. Aussi bien au Yagour qu'à Warzazat, des pierres gravées servent de murets aux azibs et "une dalle portant des gravures de grandes vaches de plus de 5 mètres est utilisée par les habitants du coin, comme aire à battre » (Searight, 2003).

\section{La proximité de deux pôles touristiques de renommée internationale : la ville de Marrakech et la vallée de l'Ourika.}

À son débouché en plaine à hauteur de la petite ville d'Aït Ourir, la vallée du Zat se situe à près de 37 kilomètres au sud-est de la ville de Marrakech, soit à moins d'une demi-heure de route. Après la petite ville du Dir des Mesfioua, la route commence progressivement à prendre de l'altitude. À hauteur du lieudit Imi n Reg (littéralement bouche de la plaine), à environ 8 kilomètres au sud d'Ait
Ourir, une bretelle permet de remonter sur sa droite la vallée du Zat jusqu'au centre de Larbaa Tighdouine sur près de 16 kilomètres. Nettement moins bien entretenue que celle de Ouarzazate, celle-ci est peu fréquentée, surtout par les autocars et les camions grande longueur. Or, elle a le mérite de relier le centre de Tighdouine à la ville de Marrakech en un peu moins d'une heure.

Cette proximité est d'autant importante que Marrakech est à la fois la capitale touristique du Maroc et le point de départ de nombreux voyages et excursions à destination soit de son arrière-pays montagneux, soit d'Agadir par le Tizi N'Machou, de Taroudant par le Tizi N'Test et de Ouarzazate par le Tizi N'Tichka. Le nombre d'agences de voyages spécialisées dans le tourisme du Grand Sud et de la Montagne est ici impressionnant.

Cependant, si les vallées de l'Ourika et d'Asni semblent avoir pleinement profité de la proximité de Marrakech, la vallée du Zat est loin d'en tirer parti. Une seule raison à cela, à notre avis : la faible médiatisation de cette dernière, l'accent ayant été mis, depuis le protectorat, essentiellement sur les vallées périphériques du Parc national du Toubkal, lui-même centré sur le massif du même nom!

Par ailleurs, la vallée du Zat, bien que voisine de la très célèbre vallée de l'Ourika dont elle n'est séparée que par le plateau du Yagour, ne semble pas avoir tiré profit de ce voisinage.

Plusieurs raisons à cela :

- d'abord, le fait que l'Ourika ait surtout développé un tourisme d'estivage pour les nationaux et d'excursion pour les étrangers, qui s'est traduit par la construction, par certains riches citadins marocains, de nombreuses résidences secondaires et, par les promoteurs touristiques, des premières structures d'accueil pour touristes étrangers ;

- ensuite, le fait que le renom de l'Ourika soit essentiellement lié à la station de ski de l'Oukaimeden, surtout après l'ouverture, en 1965, de la nouvelle route par la vallée affluente des Ait Lqaq qui permet l'accès au massif et au Parc national du Toubkal.

Cela est d'autant vrai que l'Ourika, en monopolisant les rapports avec Marrakech en matière de tourisme, a fait ombrage sur les autres vallées du Haut Atlas occidental. 
Fortement médiatisée et intensément commercialisée aussi bien à l'extérieur qu'à l'intérieur du pays, celle-ci a fini par fortement occulter les autres vallées. À la fois pour les touristes et pour les promoteurs, seule la vallée de l'Ourika et, secondairement, celle d'Asni, ont quelque chose à offrir en matière de tourisme, probablement en raison de leur similitude, toute relative d'ailleurs, avec les stations périphériques du mont Blanc, en France.

Ce n'est que grâce à la GTAM, lancée à la suite du projet Tabant en 1983 pour relier, encore une fois, le massif du Toubkal à la vallée des Ait Bougmez dans le Haut Atlas central, que la vallée du Zat a été intégrée à l'activité touristique haut atlasique: les randonneurs, en descendant l'Assif N'oufoughal, s'arrêtent à Azgour sur l'oued Zat, le plus souvent pour se reposer et manger à midi, avant de monter à Warzazat où ils bivouaquent. Après une visite, le plus souvent express, au site des gravures rupestres du même nom, ces derniers quittent la vallée à hauteur du Tizi N'Rhellis pour accéder à la vallée de l'Ourika et, par delà, rejoindre le Toubkal, Imlil et Marrakech. II s'agit là d'un début d'intégration qui a besoin, à notre avis, d'être fortement appuyé en renforçant les rapports entre les deux vallées dans le cadre d'un tourisme moins sélectif que par le passé.

\section{Une vallée encore aujourd'hui faiblement ouverte au tourisme}

La mise en tourisme de la vallée du Zat ne fait que commencer. Découverte à la suite de l'exploitation de la source minérale de Sidi El Wafi, tel qu'il a été dit ci-dessus, celle-ci n'est fréquentée que par un nombre encore relativement réduit de curistes et de randonneurs étrangers et des nombreux travailleurs émigrés qui reviennent rendre visite à leur famille. Une fréquentation qui n'a donné lieu, jusqu'à ce jour, qu'à un parc réduit de structures d'accueil et ce, en dépit de l'existence d'un nombre relativement important d'acteurs locaux prêts à jouer le rôle de pionniers dans le domaine du tourisme.

\section{Pratiques et fréquentation touristiques actuelles dans la vallée du Zat}

À la différence des vallées de l'Ourika et d'Asni dont la gamme des pratiques est sensiblement large (ski, villégiature, randonnée, ascension, écotourisme, pèlerinage), la vallée du Zat n'attire encore aujourd'hui que les adeptes de la thérapie des eaux minérales, les émigrés en visite dans leur famille et les excursionnistes de fin de semaine et des jours de repos. Très largement dominés par les nationaux, les "curistes " regroupent à la fois des malades et des non-malades. Des jeunes, des femmes et des hommes de tous âges et de tous milieux rendent visite à la source, attirés, le plus souvent, par la curiosité de découvrir "quelque chose » qui a fait beaucoup de bruit autour d'eux, surtout pendant les premières années. En été, les visiteurs deviennent étonnement nombreux - facilement près d'un demi-millier par jour, pendant les mois de juillet et août !!! - et, aux curistes, s'ajoutent les Marocains Résidants à l'Étranger (MRE), les jeunes citadins de tout bord (lycéens et étudiants) en quête de dépaysement et d'évasion et les estivants citadins à la recherche de la fraîcheur. En dehors de l'été, la fréquentation est assurée essentiellement par les excursionnistes nationaux, surtout marrakchis, de fin de semaine et des jours fériés.

Contrairement aux amateurs de Laouina, les émigrés partis travailler en dehors de la vallée, le plus souvent en ville ou dans le Souss, ont tendance à revenir auprès de leur famille, surtout en été, pour les vacances et pendant les grandes fêtes religieuses. Parce qu'ils ont fait quelques économies, ces revenants de quelques jours habillés "moderne» (casquette, jean, air max, blouson), semblent avoir acquis des pratiques quasiment similaires à celles des touristes, surtout nationaux: ils dépensent beaucoup plus que leurs semblables restés sur place, fréquentent plus souvent « le village » de Larbaa en voie d'urbanisation et n'hésitent pas à visiter Laouina en touristes par imitation des citadins.

Par ailleurs, en dehors des catégories de visiteurs nommées ci-dessus, la vallée est «traversée » par un nombre de plus en plus grand de touristes étrangers dans le cadre de la GTAM. Reliant la vallée des Ait Bougmez à l'est à celle d'Asni au pied du Toubkal à l'ouest, la GTAM transite par la vallée du Zat à hauteur d'Azgour et de Warzazt. De passage, les randonneurs ne retiennent de cette dernière que les noms d'Azgour, de Warzazt et du plateau du Yagour, soit un itinéraire est-ouest qui relie les vallées du Rdat et de l'Ourika le long de l'Assif n Oufoughal par le Tizi N'Rhellis. II s'agit là, en définitive, de pratiques touristiques fort classiques, d'un côté, et d'une fréquentation encore très en deçà des potentialités touristiques de la vallée du Zat, de l'autre; des indicateurs dont l'impact sur les structures d'accueil ne peut être que limité.

\section{Des structures d'accueil quasiment inexistantes à ce jour}

La particularité des pratiques touristiques qui se développent dans la vallée du Zat ne peut contribuer qu'à la création de structures d'accueil de bas niveau. En effet, si les randonneurs et les émigrés de retour au pays sont totalement pris en charge, d'aucuns par les agences de voyages qui leur assurent aussi bien l'accompagnement, l'hébergement que la bouffetance, et les autres par leur famille, seuls les excursionnistes et les curistes peuvent être à la recherche de structures d'accueil qui leur permettent de séjourner dans la vallée. La réponse à cette demande a engendré un important développement du centre de Larbaa. La construction de chambres ou de maisons destinées à la location s'est fortement développée au cours des premières années d'exploitation de la source de Sidi EI Wafi. Un semblant d'hôtel de quinze chambres a même été édifié à Larbaa par un ancien émigré de retour au pays !

Un peu partout, des boutiques et des cafés-restaurants voient le jour en vue de répondre aux besoins des touristes en produits de tout genre (viande, légumes et fruits, pâtisserie) et la commune a aménagé un parc de stationnement automobile. Cependant l'éloignement, quoique très relatif, de Laouina par rapport au centre de Larbaa, pose problème, en dépit de l'émergence d'une activité jusque-là inconnue dans la vallée: le portage à dos de mulets des visiteurs.

Pour résoudre ce problème, les propriétaires des terrains voisins de la source ont commencé à héberger les touristes dans des tentes qui se sont transformées, d'abord en huttes, puis en cabanons, puis enfin en résidences construites en parpaing, le tout dans un manque total d'ordre d'esthétique et de goût, l'objectif principal ayant été d'héberger le maximum de touristes au moindre coût et ce, en raison de la modicité des moyens aussi bien des clients que des promoteurs euxmêmes. Par manque de moyens financiers, mais aussi parce que mal conseillés et entièrement laissés à eux-mêmes, ceux-ci ont fini par tout rater. 
Se donnant pour objectif de remédier à cet état de fait, des initiatives impulsées de l'intérieur voient le jour : elles consistent à proposer aux touristes des structures d'accueil de qualité susceptibles à la fois de satisfaire une demande touristique de plus en plus exigeante et de préserver la beauté de l'environnement de la vallée. C'est dans ce cadre que s'inscrit, par exemple, la construction par l'Association des Amis du Zat de trois gites ruraux aux douars de Tizirt, de Warzazt et d'Aït Ali, dans le respect total de l'architecture et des savoir-faire locaux.

Ces gîtes, réalisés avec la participation active des villageois, s'adressent, en priorité, aux amateurs de la randonnée douce (pédestre ou équestre) et aux amoureux de la nature et de l'écotourisme ${ }^{1}$, des produits nouveaux qui permettent de sortir des sentiers battus et de proposer d'autres formes de tourisme dont la dynamisation doit être assumée, en priorité, par les acteurs locaux de développement (populations, associations et collectivités locales).

\section{Conclusion}

Le tourisme, tel qu'il est pratiqué aujourd'hui dans les vallées dites touristiques de l'Ourika et d'Asni, rapporte peu, de l'avis des habitants eux-mêmes. Pire encore, il ne fait qu'accentuer les inégalités entre les familles au sein d'un même douar et entre les douars d'une même vallée. Les vrais bénéficiaires de la manne touristique, surtout internationale, sont les hôtels et les hôtels-restaurants capables de traiter avec les voyagistes, mais dont la propriété aussi bien que la gestion échappent totalement à la vallée.

Loin donc de servir de facteur de développement pour les vallées du Haut Atlas, le tourisme est en train de les «vider » des éléments les plus actifs de leurs habitants par le biais de l'émigration en ville, soit carrément à l'étranger, pour les plus « chanceux » d'entre eux. C'est donc à la fois pour en limiter les effets pervers de tout genre et pour en améliorer les retombées positives tant sur les conditions de vie des populations locales que sur l'environnement de ces vallées, que le tourisme en zone de montagne doit, à notre avis, être :

- un tourisme doux et discret, autrement dit un tourisme de petits groupes ou carrément de familles, et non un tourisme de masse, provocateur et arrogant;
- un tourisme solidaire et équitable, soit un tourisme de partage et non de frustration et d'enrichissement d'une minorité et, par conséquent, un tourisme qui permet à ceux qui le pratiquent de participer au développement des douars et des pays qui les accueillent et ce, en s'impliquant dans la réalisation des actions de lutte contre l'analphabétisme, des actions d'approvisionnement en eau potable, de petite hydraulique, de soutien à la scolarisation de la «fille rurale »... par exemple;

- un tourisme respectueux de l'environnement tant naturel que culturel et humain des espaces haut atlasiques, capable de préserver aussi bien la beauté des sites et des paysages que la cohésion des communautés villageoises des vallées ou des pays du Haut Atlas de Marrakech;

- un tourisme fondé sur l'écotourisme, sur le tourisme de découverte et d'échange, susceptible de rapprocher visiteurs et visités et, à travers eux, de favoriser le dialogue des cultures et des civilisations, combien indispensable pour le rapprochement des peuples et des nations;

- un tourisme fortement intégré à l'économie locale, autrement dit un tourisme capable de dynamiser les autres secteurs de l'économie des vallées haut atlasiques appelés à répondre, localement, à la demande des touristes en produits agricoles et artisanaux de tout genre (fruits et légumes, viande, œufs, volaille, pain, poteries, huile, noix, tapis...), mais aussi en commerces et services des plus diversifiés ;

- un tourisme, enfin, dans lequel les associations locales et les organisations non gouvernementales internationales sont appelées à jouer un grand rôle, le tourisme associatif ayant toujours eu pour ultime objectif d'aider à l'épanouissement des populations les plus démunies et au développement des contrées les plus défavorisées (Bellaoui, 2001).

Des conditions qui sont, si elles sont réunies, à même de faire du tourisme un véritable levier de développement des zones de montagne au Maroc, en général, et de la vallée du Zat, en particulier.

Ahmed Bellaoui est enseignant-chercheur à l'Université Cadi Ayyad, spécialisé en géographie-aménagement. Il est responsable du cours "Géographie du tourisme » à l'École doctorale internationale du Tourisme.

\section{Notes}

1 En dehors de l'Association des amis du Zat, il existe dans la vallée d'autres associations qui se donnent pour objectif de promouvoir le tourisme dans la vallée; mais leur action demeure relativement modeste. II s'agit des associations de Sidi EI Wafi, la section locale des Maisons d'Hôtes du Sud et de Tawada. Deux promoteurs privés de Marrakech viennent d'aménager des maisons d'hôte rurales aux douars de Timzellit et d'Ighalen à proximité du centre d'Arbia Tighdouine.

\section{Bibliographie}

Bellaoui, Ahmed (1989), "Les Pays de l'Adrar N'Dern, étude géographique du Haut Atlas de Marrakech", thèse de Doctorat d'État ès lettres, Université de Tours.

Bellaoui, Ahmed (1990), L'agriculture dans le Haut Atlas de Marrakech entre passé et présent, Marrakech, Journée d'étude organisée par le département de Géographie, Faculté des Lettres et des Sciences Humaines, 8 mars. Université de Marrakech.

Bellaoui, Ahmed (1992), "Le Haut Atlas occidental, un espace très fortement humanisé ", Revue de la Faculté des Lettres et des Sciences Humaines, $n^{\circ} 7$, Université de Marrakech.

Bellaoui, Ahmed (1994), « Le Dir septentrional de l'Adrar N'Dern, un espace de transition en pleine mutation ", Études méditerranéennes, n 18, Poitiers et Fascicule de recherches n 26, URBAMA, Tours.

Bellaoui, Ahmed (2001), Pour un tourisme de montagne doux, discret, intégré et de qualité. Journée d'étude organisée à Ouirgane par la Chambre de commerce et de services de Marrakech, juillet.

Bellaoui, Ahmed (2004), "Le tourisme dans le Haut Atlas de Marrakech : une activité qui, pour être durable, demande à être autrement organisée et réglementée ", Le tourisme durable, réalités et perspectives marocaines et internationales, Publication du CRCN et de l'EDIT, Faculté des Lettres et des Sciences Humaines, Marrakech, Université de Marrakech.

Boujrouf, Saïd (1994), «Un nouveau produit pour le tourisme national de montagne: le thermalisme à l'Arbia Tighdouine (Haut Atlas de Marrakech)", Revue de la faculté des Lettres et des Sciences Humaines, n 10, Université de Marrakech.

Dresch, J., et J. De Lepiney (1938), Guide a/pin de la montagne marocaine, Casablanca.

Malhomme, J. (1959), Corpus des gravures rupestres du Grand Atlas, $1^{\text {re }}$ partie, Rabat, Publication du service des Antiquités du Maroc.

Searight, S. (2003), "Gravures rupestres du Haut Atlas. Montagnes Marocaines ", Revue du Club alpin français, automne no 11.

Office national marocain du Tourisme (1995), La grande traversée des Atlas marocains, Rabat, Guide de renseignements pratiques. 